\title{
Surgical Treatment of Advanced Heart Failure- Left Ventricular Assist Device Implantation and Adjuvant Tricuspid Valve Repair
}

\begin{abstract}
Background: We present the case of a successful surgical treatment of terminal heart failure. The patient has had left ventricular assist device implantation done in order to maintain vital parameters and bridging to cardiac transplantation period. Tricuspid valve repair has been done at the same time to correct the tricuspid regurgitation and indirectly prevent deterioration of RVF.

Case presentation: Left ventricular assist device Heart Mate 3 was implanted with tricuspid valve reconstruction in an 53-year-old man with a history of cardiomyopathy. At the time of admission and despite medical therapy, he had decompensated heart failure, low cardiac output syndrome, impending organ failure and consequent end-organ dysfunction. His symptoms had progressively worsened. After adequate preoperative preparation the patient was operated. On a beating heart tricuspid valve annuloplasty with semi-rigid ring preceded pump implantations. The right atriotomy was closed and the Heart Mate 3 device was implanted without aortic clamping in order to provide support to the left ventricular as a bridge to transplantation. The inflow cannula device was installed over the top of the left ventricle. The power cable was placed through the skin in was the form of double tunneling and connected to the controller and the energy source. Finally, a graft sewn to the aorta.
\end{abstract}

Conclusion: Persistent tricuspid regurgitation contributes to morbidity and mortality following LVAD implantation. An effective solution without increasing operative mortality at the time of LVAD implantation is provided by tricuspid ring annuloplasty.

Keywords: LVAD; Heart Mate III; Tricuspid valve regurgitation

Case Report
Volume 9 Issue 5 - 2017
Duško Terzić1 ${ }^{1 *}$, Emillija Nestorović ${ }^{1}$,
Svetozar Putnik ${ }^{1}$, Dragan Ivanisevic ${ }^{1}$, Jelena
Cumic $^{2}$ and Miljko Ristić1
${ }^{1}$ Clinic for Cardiac Surgery, Clinical Center of Serbia, Serbia
${ }^{2}$ Center for Anesthesiology and Reanimation, Clinical Center of
Serbia, Serbia
*Corresponding author: Duško Terzić, Clinic for Cardiac
Surgery, Clinical Center of Serbia, Dr. Koste Todorovića 8,
11000 Beograd, Serbia, Tel: +381668301966; Email:
terzic.dusko@gmail.com
Received: July 27, 2017 | Published: August 31, 2017

\section{Background}

Cardiac transplantation is the treatment of choice in patients with end-stage heart failure. Insufficient number of donors in all health systems resulted in the need for intensive development of devices for mechanical circulatory support. Right ventricular failure (RVF) combined with major tricuspid insufficiency among the group of patients who are candidates for the left ventricular assist device (LVAD) represents a significant risk factor in terms of having a successful outcome of surgical treatment of cardiac insufficiency.

This paper presents the case of a successful surgical treatment of terminal heart failure in a 53-year old male patient who had LVAD implant surgery as a bridge to transplant with adjuvant tricuspid valve repair.

\section{Case Presentation}

Left ventricular assist device Heart Mate 3 and tricuspid valve reconstruction was implanted in an 53-year-old man with a history of cardiomyopathy of unknown cause, which was diagnosed approximately 4 years before he was admitted to our hospital. At the time of admission, despite medical therapy, he had decompensated heart failure, low cardiac output syndrome, impending organ failure and consequent end-organ dysfunction. His symptoms had progressively worsened. He was treated in our hospital with inotropic medications, intravenous diuretics, anti arrhythmic drugs. Patient was categorized as NYHA class IV, INTERMACS class II.

An echocardiogram on admission revealed the left ventricle markedly dilated $(7.3 / 6.9 \mathrm{~cm})$, burdened by volume (EDV 276 $\mathrm{ml}$ ), normal wall thickness, with reduced overall systolic function - ejection fraction (EF) of $9 \%$ by Simpson, $10-15 \%$ by Biplane and $10 \%$ by Teiholc. The mitral apparatus of preserved morphology, with moderate functional mitral regurgitation $2+$. The right heart chamber was of increased size $(4.2 \mathrm{~cm})$. Through the tricuspid valve registered serious tricuspid valve regurgitation (TR) of $4+$, the eccentric stream directed towards the lateral wall of the DP. Tricuspid annulus width of $4.3 \mathrm{~cm}$. Indirectly estimated pressure in the right heart of $31 \mathrm{mmHg}$. Both sides were with pleural effusion. As cites was in the peritoneal cavity. 
After adequate preoperative preparation the patient was operated. The procedure was performed through a full median sternotomy. With an appropriate activated clotting time $(>400$ seconds), patients were placed on cardiopulmonary bypass. On a beating heart tricuspid valve annuloplasty with semi-rigid ring preceded pump implantations. The right atriotomy was closed and the LVAD implanted. The Heart Mate 3 device was implanted without aortic clamping in order to provide support to the left ventricular as a bridge to transplantation. The inflow cannula device was installed over the top of the left ventricle. The power cable was placed through the skin in was the form of double tunneling and connected to the controller and the energy source. Finally, a graft sewn to the aorta. Unlike Heart Mate 2 device where it is necessary to form a tissue pocket to place the device, HM3 device was applied fully intrapercardially. The intervention passed without complications (Figure 1).

The postoperative course at the clinic for cardiac surgery also regular. The pump speed was set at $4800 \mathrm{rpm}$, PF 3,4, PP 3,2, PI 3,3 , Hct 27.Stable LVAD parameters and data from the controller
Auscultation shows cardiac rhythmic action, clear sound of LVAD pump without variations in intensity. Laboratory and radiological analysis within the reference values (Figure 2). The echocardiography at discharge showed the left ventricle increased in size, smaller in (EDD 6.6, ESD $5.7 \mathrm{~cm}$ ), normal wall thickness. A slight MR 1+ is recorded into the left atrium with dimensions of $4.6 \mathrm{~cm}$. The right ventricle was of smaller dimensions comparison to the preoperative findings of $2.6 \mathrm{~cm}$, better systolic and longitudinal functions. There was still the presence of TR 1-2+ of eccentric stream directed towards the DP wall. A mild PR 1+ was recorded. The input and output cannulas of device were with excellent colors with the flow rate. The patient and his family were educated regarding the device's functioning (battery charging, alarm registration) and the hygiene of the output area of the drive line. The patient no showed symptoms of heart failure and was physically active. After one month the patient was discharged. On follow-up examination one and two months after discharge, LVAD parameters and data on controller were stable. The LVAD alarms were not registered. The patient was put on the list for a heart transplant.

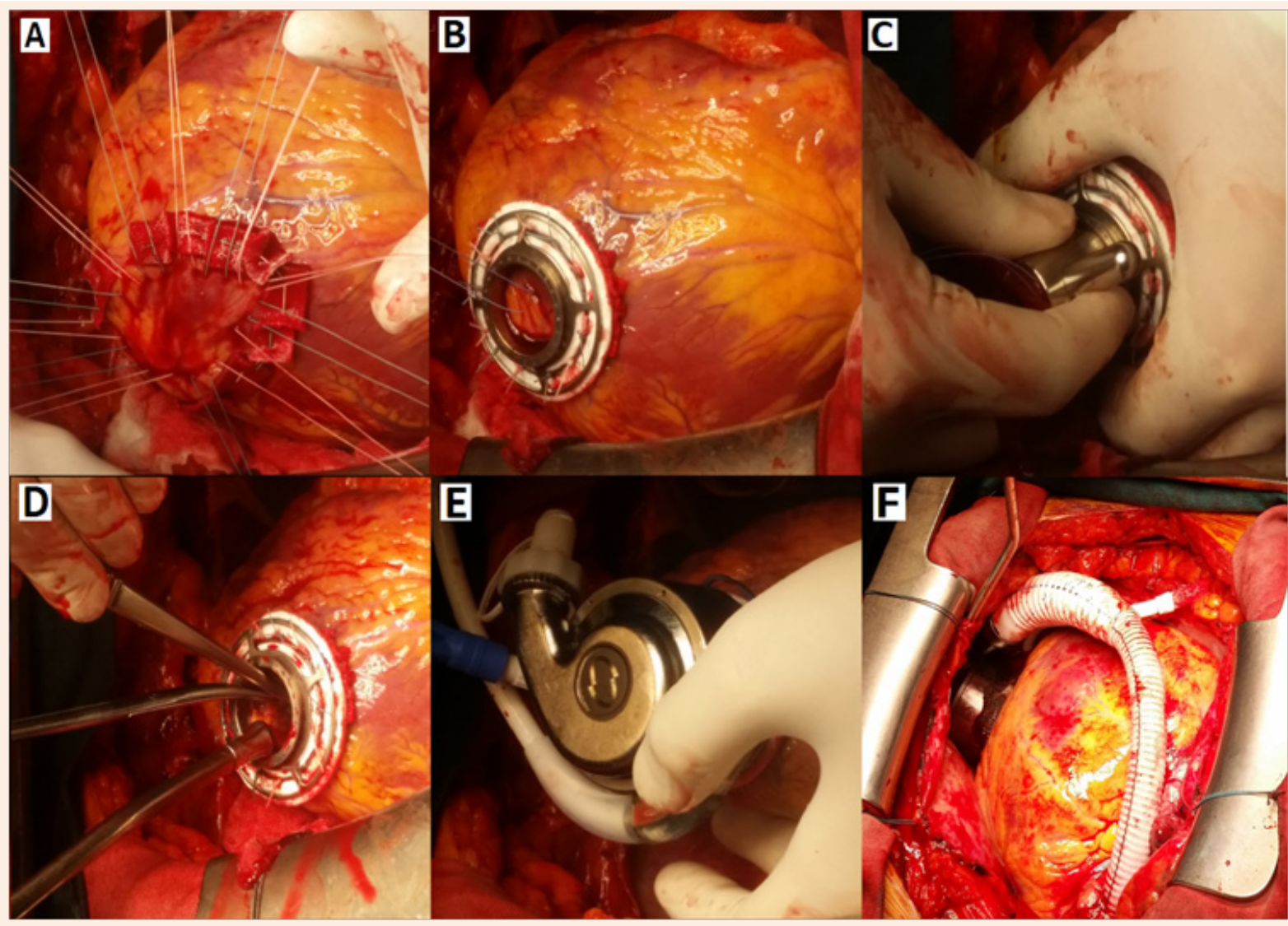

Figure 1: LVAD Heart Mate 3 implantation

a, b) sewn ring at the left ventricle

c, d) left ventricle open at the top

e, f) connected pump with heart in pericard cavity 


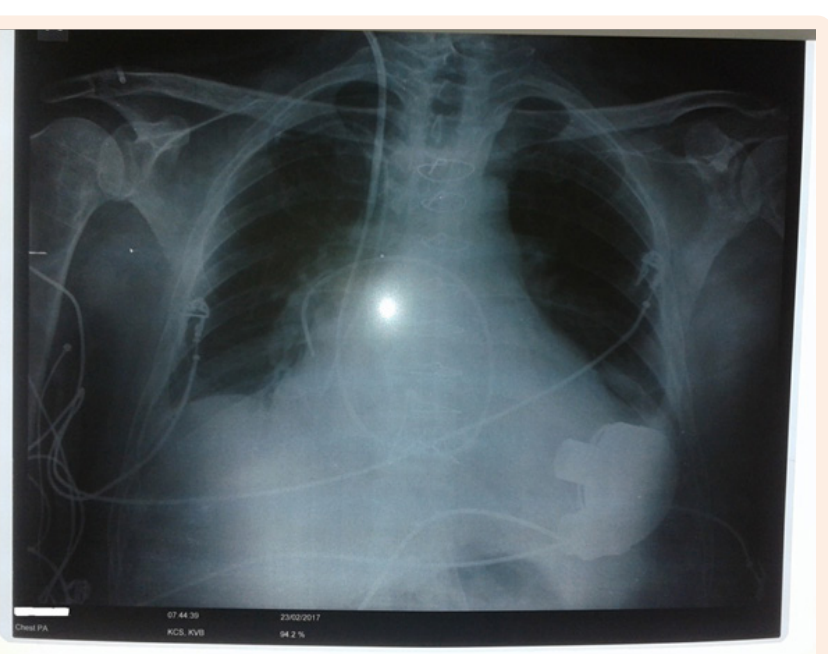

Figure 2: Radiografic findings after LVAD Heart Mate 3 implantation and tricuspid valve reconstruction.

\section{Discussion}

Implantable mechanical circulatory devices, such as left ventricular assist device (LVAD), have emerged as a relevant option for improving quality of life and survival in patients with end-stage heart failure. The most common indications include bridge to transplant (BTT), destination therapy (DT), bridge to recovery (BTC), and bridge to candidacy (BTR) $[1,2]$.

Besides being a life-saving treatment, LVADs presently enable patients with severe HF to survive and live considerably longer with agreeable quality of life. Therefore, long-term LVAD implantation has become a valuable option to cardiac transplantation for treating end-stage HF. According to the studies, post-transplant survival at one, two, five and 10 years is approximately 90, 80, 70 and 50 per cent. Therefore, the need for cardiac transplantation as a first-line replacement therapy can be reduced by extended post-transplant survival in patients receiving BTT [3].

A new centrifugal-flow device known as HeartMate 3LVAD uses full magnetic levitation that enables wide and consistent flow paths and an artificial pulse designed for enhanced hemocompatibility. Utilizing fully magnetically levitated rotor with frictionless movementthe LVAD Heart Mate 3 prevents heat generation and wear of the moving component. Its internal surfaces textured with titanium microspheres stimulate adhesion of the patient's cells to develop a tissue lining. The device is designed to provide longterm hemodynamic support in patients with advanced refractory left ventricular heart failure either for temporary support such as a bridge to transplantation, myocardial recovery, or as destination therapy. [4].

Nevertheless, even in spite of the favourable results, right heart failure (RHF) or progressive decline of right ventricular function still remains one of major problems amongst the group of patient candidates for LVAD implant. Furthermore, there's a possibility of RHF leading to the weakened flow of LVAD, difficulty in weaning from cardio-pulmonary bypass (CPB), decreased tissue perfusion and multi-organ failure that are connected with increased morbidity and mortality. Those patients with heart failure who are candidates for LVAD implantation may show a wide clinical spectrum of preoperative right ventricle (RV) dysfunction, starting from being relatively asymptomatic to developing fulminant right ventricular failure [5].

Patients with implanted LVAD who develop RVF have a higher incidence of post-operative bleeding, thrombo-embolic events and multi organ dysfunction. It is from these reasons that preoperative evaluation functions of the right ventricle and the subsequent RVF treatment is one of the crucial factor for a successful outcome after installing the post implanted LVAD. The values of central venous pressure, pulmonary capillary wedge pressure and secondary pulmonary hypertension are predictive of RVF after LVAD implantation [6].

Primary imaging modality for monitoring cardiac function in heart failure patientsis still echocardiography. Cardiac magnetic resonance imaging (MRI) has recently emerged as the gold standard but is not routinely used. RV assessment includes an evaluation of RV shape, size, volume, wall thickness, deviation of the septum towards the left or the right ventricle, evaluation of the dilatation of right atrium, right ventricle, of main pulmonary artery and branches, inferior vena cava dilatation and its respiratory alterations, tricuspid valve annulus, and tricuspid regurgitation (TR) degree [1,7]. Postoperative may be worsened by TR due to an intra ventricular septum shift to the left. Secondary tricuspid regurgitation is common in patients with RVF undergoing LVAD implantation, so that the tricuspid valve repair in the same act with the implantation is associated with a reduction in postoperative RVF. The decision to perform tricuspid valve repair during LVAD implantation correlates to: TV annulus $>40 \mathrm{~mm}$ and moderate or severe TR. In the event of corrected TR, it may help venous flow and renal perfusion and improve postoperative morbidity [7].

LVADs and Heart Mate 3 may also reversibly or irreversibly deteriorate the function of the right ventricle in the initial period after implantation of the device due to a greater cardiac output, which causes a greater venousflow and, consequently, load (preload) of the right ventricle.RVF treatment after LVAD implantation includes inotropic support, reduction of pulmonary vascular resistance, inhalation of nitric oxide, and in more severe cases, the application of extra corporal membrane oxygenator (ECMO), or the implantation of right ventricular assist device $\operatorname{RVAD}[7,8]$.

The patient has had LVAD implantation done in order to maintain vital parameters and bridging to cardiac transplantation period. Tricuspid valve repair has been done at the same time to correct the tricuspid regurgitation and indirectly prevent deterioration of RVF.

\section{Conclusion}

Persistent tricuspid regurgitation contributes to morbidity and mortality following LVAD implantation. An effective solution without increasing operative mortality at the time of LVAD implantation can be provided by tricuspid ring annuloplasty. 


\section{References}

1. Argiriou M, Kolokotron SM, Sakellaridis T, Argiriou O, Charitos C et al. (2014) Right heart failure post left ventricular assist device implantation. J Thorac Dis 6 Suppl 1: S52-S59.

2. Kormos RL, Teuteberg JJ, Pagani FD, Russell SD, John R, et al. (2010) Right ventricular failure in patients with the HeartMate II continuous-flow left ventricular assist device: incidence, risk factors, and effect on outcomes. J Thorac Cardiovasc Surg 139(5): 1316-1324.

3. Takeda K, Takayama H, Kalesan B, Uriel N, Colombo PC, et al. (2014) Long-term outcome of patients on continuous-flow left ventricular assist device support. J Thorac Cardiovasc Surg 148: 1606-1614.

4. Netuka I, Sood P, Pya Y, Zimpfer D, Krabatsch T, et al. (2015) Fully Magnetically Levitated Left Ventricular Assist System for Treating Advanced HF: A Multicenter Study. J Am Coll Cardiol 66(23): 25792589.
5. Kukucka M, Potapov E, Stepanenko A, Weller K, Mladenow A, et al. (2011) Acute impact of left ventricular unloading by left ventricular assist device on the right ventricle geometry and function: effect of nitric oxide inhalation. J Thorac Cardiovasc Surg 141(4): 1009-1014.

6. Kalogeropoulos AP, Kelkar A, Weinberger JF, Morris AA, Georgiopoulou VV, et al. (2015) Validation of clinical scores for right ventricular failure prediction after implantation of continuousflow left ventricular assist devices. J Heart Lung Transplant 34(12): S1053-2498.

7. Meineri M, Van Rensburg AE, Vegas A (2012) Right ventricular failure after LVAD implantation: prevention and treatment. Best Pract Res Clin Anaesthesiol 26(2): 217-229.

8. Haneya A, Philipp A, Puehler T, Rupprecht L, Kobuch R, et al. (2012) Temporary percutaneous right ventricular support using a centrifugal pump in patients with postoperative acute refractory right ventricular failure after left ventricular assist device implantation. Eur J Cardiothorac Surg 41(1): 219-223. 